\title{
Special issue on multimedia fog/edge computing systems
}

\author{
Yong $\mathrm{Jin}^{1}$ • Daniele D'Agostino ${ }^{2}$ - Nadjib Achir ${ }^{3}$ - James Nightingale ${ }^{4}$. Jiacun Wang ${ }^{5}$
}

Published online: 3 January 2020

(c) Springer-Verlag GmbH Germany, part of Springer Nature 2020

Technological advancements have enabled emerging multimedia applications requiring powerful devices with realtime capabilities, and advanced computation and control. However, many end devices are characterized by limited resources that pose significant challenges to these devices in providing multimedia services effectively. Though, the traditional cloud computing paradigm as the de facto centralized big data processing platform may not be efficient enough to support many emerging multimedia applications with QoS/ QoE/QoI constraints. Nowadays, fog/edge computing has become a promising paradigm for multimedia systems. The basic idea is to leverage a multitude of collaborative end devices and near-user infrastructures to carry out a substantial amount of computation, storage, and communication tasks, enabling low latency, energy efficiency as well as agile computation augmenting services for multimedia. Despite the many benefits and opportunities fog/edge computing offers, there are several research and technical challenges that need attention from the research community. Some of these challenges include: how to design an efficient fog/

Yong Jin

jinyong@cslg.edu.cn

Daniele D'Agostino

dagostino@ge.imati.cnr.it

Nadjib Achir

nadjib.achir@univ-paris13.fr

James Nightingale

james.nightingale@uws.ac.uk

Jiacun Wang

jwang@monmouth.edu

1 Changshu Institute of Technology, Changshu, China

2 Institute for Applied Mathematics and Information Technologies, National Research Council of Italy, Via Marini 6, I-16149 Genoa, Italy

3 University of Paris 13, 93430 Villetaneuse, France

4 University of the West of Scotland, Glasgow, UK

5 Computer Science and Software Engineering Department, Monmouth University, West Long Branch, NJ, USA edge computing-based multimedia computing framework, how to make multimedia systems more intelligent, how to achieve the efficient multimedia data processing and distribution with edge/fog computing, and how to design a fog/ edge computing assisted service enhancement mechanism.

The paper "QoI-Aware Incentive for Multimedia Crowdsensing Enabled Learning System" by Yiren Gu, proposed a Quality-of-Information aware incentive mechanism in multimedia crowdsensing, with the objective of promoting the growth of an initial training model. The paper "A Novel Automatic Image Segmentation Method for Chinese Literati Paintings Using Multi-view Fuzzy Clustering Technology" by Jie Zhang et al. invested a new robust multi-view (M-V) fuzzy clustering algorithm for image segmentation of Chinese literati paintings to achieve effective decomposition and extraction of ancient paintings. The paper "ThreeDimensional Laser Image Filtering algorithm based on Multi-source Information Fusion and adaptive Offline Fog Computing" by Wei Yan, proposed an improved adaptive mean shift image filtering algorithm based on the traditional mean shift filtering algorithm to improve the image processing accuracy and speed of laser three-dimensional imaging system, effectively filter the noise in the image, and effectively optimize the processing speed and image accuracy. The paper "Fr'echet Mean based Grassmann Discriminant Analysis" by Hongbin Yu et al. proposed Fr'echet Mean based Grassmann Discriminant Analysis algorithm to implement the videos (or image sets) data dimensionality reduction and clustering task. The paper "Person-Independent Facial Expression Recognition Method Based on Improved Wasserstein Generative Adversarial Networks in Combination with Identity-Aware" by Caie Xu et al. proposed a person-independent recognition method based on Wasserstein generative adversarial networks for micro-facial expressions, where a facial expression recognition network and a facial identity recognition network are established to improve the accuracy and robustness of facial expression recognition via inhibition of intra-class variation. The paper "3D Point Cloud Registration Denoising Method for Human Motion Image Using Deep Learning Algorithm” by Qidong Du, 
proposed a 3D point cloud registration denoising method for human motion image using depth learning algorithm in complex scenes. The paper "Oriented Grouping-Constrained Spectral Clustering for Medical Imaging Segmentation" by Kaijian Xia, proposed an oriented grouping-constrained spectral clustering method to deal with medical image segmentation problems.
We would like to thank all reviewers who have helped in the paper review process, and the authors for their contribution and efforts to complete the papers with very high quality. Finally, we express our gratitude to Dr. Thomas Plagemann for initiating this special issue and inviting us to undertake this rewarding activity. 